\title{
DESARROLLO DE UN INVERSOR 12 VCC A 220 VCA CON SITEMA DE SENSADO DE CARGA PARA MARCHA A DEMANDA
}

Víctor Marder ${ }^{1}$, Carlos Cadena ${ }^{2}$, Víctor Toranzos ${ }^{1}$

\section{RESUMEN}

En el presente trabajo se detallan los circuitos componentes del inversor, con una breve descripción de su funcionamiento y criterios básicos de diseño. Este tipo de inversores tiene la particularidad de minimizar el autoconsumo mientras no se conecte ninguna carga, generando solamente un tren de pulsos cada dos segundos. Cuando una carga es conectada el sistema se enciende y opera normalmente hasta que la carga se desconecte. Esta tecnología es adecuada para uso fotovoltaico autónomo.

Palabras claves: fotovoltaica autónoma, marcha a demanda, conversión de energía.

\section{INTRODUCCIÓN}

La conversión de energía fotovoltaica es altamente modular, las instalaciones pueden variar entre milivatios para productos electrónicos (carga de celulares, relojes, linternas, etc) a megavatios en el caso de los sistemas conectados a red. Esto da lugar a una amplia variedad de aplicaciones de la energía fotovoltaica $(\mathrm{FV})$. Es de especial interés, sobre todo en lugares sin acceso a la red eléctrica, el desarrollo de sistemas de suministro de electricidad a partir de las instalaciones $\mathrm{FV}$ de baja potencia, con la ventaja que los módulos fotovoltaicos se pueden añadir gradualmente al sistema con el fin de hacer frente al posible crecimiento de la demanda de energía. De esta forma el suministro de electricidad se pueden realizar evitando una inversión inicial alta. En el contexto de la electrificación rural y para el suministro de energía de aparatos eléctricos fuera de la red, la característica de bajo mantenimiento por la ausencia de partes móviles de la energía FV es también otra ventaja considerable (Luque y Hegedus, 2011). Es de nuestro especial interés las instalaciones $\mathrm{FV}$ de baja potencia y con ello nos referimos a uno pocos kilovatios, dado que en nuestro entorno universitario se pueden hacer pequeñas contribuciones para el desarrollo de equipos nacionales o para la capacitación y entrenamiento de profesionales e idóneos en el área de fotovoltaica domiciliaria.

El desarrollo de inversores simples de onda cuadrada o senoidal modificada se encuentra bastante trillado, pudiéndose realizarlos con electrónica discreta o mediante el uso de microcontroladores.

Sin embargo en la mayoría de los proyectos electrónicos que se pueden encontrar sobre pequeños inversores, difícilmente

1- Departamento de Ingeniería, FACENA, UNNE, Corrientes, Corrientes, CP3400

2- INENCO-Departamento de Física, UNSa / e-mail: victoranzos@gmail.com 
estos dispongan de la función de marcha a demanda con sensado de carga. Esta función es común en los inversores de uso fotovoltaico dado que minimiza la corriente de autoconsumo mientras no exista carga, y permite el encendido del inversor de manera automática cuando se conecta una carga al circuito de consumo resultando en una gran comodidad para el usuario, el que apenas percibe un ligero retraso entre oprimir una tecla y ver encenderse la luz por ejemplo (hoja técnica inversor Magnum Energy).

Para esta función debe poder detectarse corrientes pequeñas, como el caso de una lampara de $10 \mathrm{~W}$ que solo tomaría $45 \mathrm{~mA}$ en 220 Vca.

\section{DESARROLLO DEL INVERSOR:}

\section{Generador de $50 \mathrm{~Hz}$ :}

El corazón del proyecto es un circuito integrado (CI) TL494 (Hoja técnica de Motorola) que es de uso generalizado en fuentes conmutadas para computadoras personales. Este CI fue concebido para su empleo a frecuencia de varias decenas de $\mathrm{KHz}$, sin embargo, es posible emplearlo en bajas frecuencias (decenas de $\mathrm{Hz}$ ).

La frecuencia de operación se fija mediante un circuito oscilador RC (RT pata 6 y CT pata 5) que para $50 \mathrm{~Hz}$ se utilizaron los valores comerciales $\mathrm{R}=120 \mathrm{Ky} \mathrm{C}=0,1 \mathrm{uF}$.

E1 CI entrega en la pata 9 y 10 dos señales rectangulares desfasadas $180^{\circ}$ que son requeridas para proveer la excitación a la etapa de potencia.

El tiempo de duración de estas señales rectangulares (ancho del pulso) se varía para obtener la tensión de salida requerida, lográndose buena regulación mediante un lazo de realimentación constituido por un circuito rectificador de onda completa y un opto-acoplador que actúa sobre la pata 4 (DTC) del CI modificando el ancho del pulso. El circuito del generador de $50 \mathrm{~Hz}$ se muestra en la figura 1.

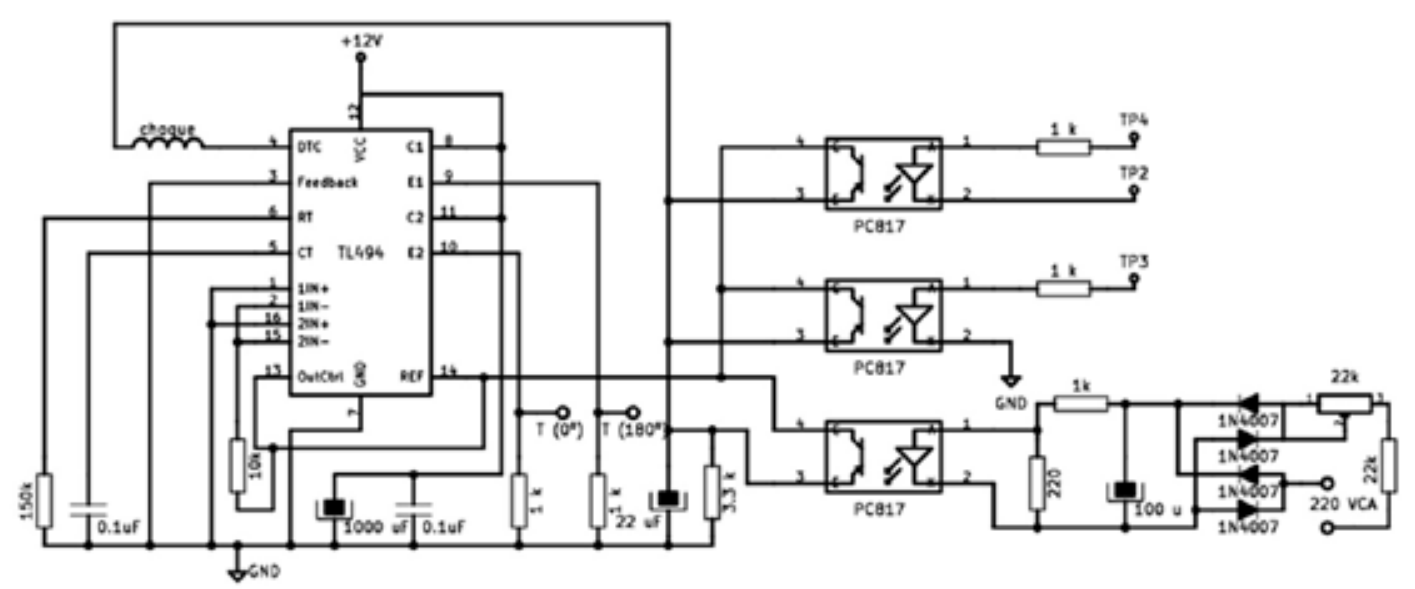

Figura 1, Circuito esquemático del generador de $50 \mathrm{~Hz}$ con modulación de ancho de pulso 


\section{Etapa de potencia:}

Esta etapa del inversor se realizó con transistores MOSFET IRFZ44 (Hoja de datos) en configuración "push-pull" y un transformador elevador que dispone de punto medio en su devanado de baja tensión, cuya relación de transformación es de 27 veces y $300 \mathrm{~W}$ potencia. A la izquierda de la figura 2 se muestra el diagrama de circuito de la etapa de potencia, donde se incluye también un sistema de absorción de sobretensiones transitorias, que podrían dañar los transistores de potencia, mediante dos diodos y un circuito RC. Además en el mismo esquema se puede apreciar una fuente auxiliar de $5 \mathrm{~V}$ constituida principalmente por un diodo zener y un mínimo de componentes periféricos.
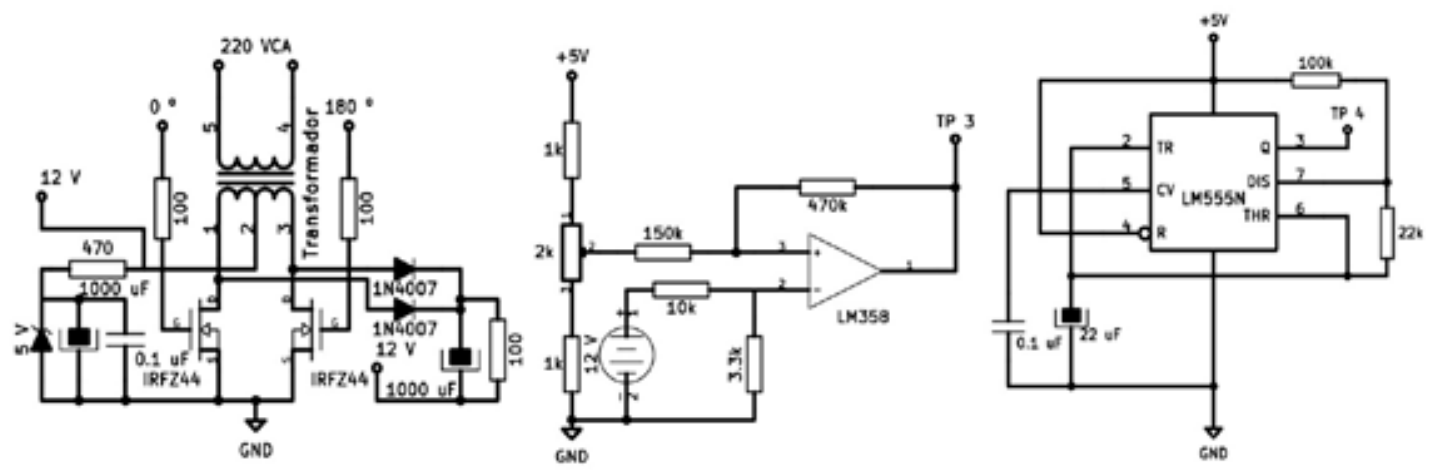

Figura 2: Circuitos esquemáticos. Izquierda: Etapa de potencia, Centro: Detector de estado de bateria, Derecha: Sistema de testeo.

\section{Circuito generador para testeo:}

Este circuito se encarga de encender el inversor en períodos de 2 segundos con un ciclo útil de un $10 \%$, que corresponde a 10 ciclos de $50 \mathrm{~Hz}$. Si en este intervalo se detecta la presencia de una carga el inversor permanece encendido hasta que la carga se desconecta. El circuito consiste en un oscilador astable realizado con un CI LM555 (Hoja de datos) y el esquema de circuito se muestra a la derecha de la figura 2 .

\section{Sensado de estado de carga de batería:}

Para alimentar este inversor se prevé el uso de una batería de plomo ácido o plomo gel, en la cual no debe permitirse su descarga si la tensión en bornes se encuentra por debajo de cierto valor, el cual suele estar fijado por arriba de los 10 voltios. Para ello se implementó un discriminador que compara una tensión de referencia, ajustable con un potenciómetro, con la tensión de batería y provee cierta histéresis (1,5 V aproximadamente) para evitar oscilaciones indeseadas. El circuito se realizó con un CI operacional LM358 y su descripción esquemática se muestra en el centro de la figura 2.

\section{Detector de carga:}

El mismo costa de dos etapas separadas galvánicamente por un dispositivo optoacoplador. En primer lugar analicemos la etapa de entrada, destinada a medir corriente y por tanto se interpondrá en serie entre la carga y el inversor. El camino para 
la corriente alterna está dado principalmente en un sentido por dos diodos 6A10 (hoja técnica de Diodes Inc.) puestos en serie y en el otro por uno solo. Como estos diodos son de juntura de silicio presentan en directa una caída de tensión de aproximadamente $0,7 \mathrm{~V}$ dando la serie una tensión de $1.4 \mathrm{~V}$, la que resulta suficiente para hacer funcionar el diodo emisor de luz (LED) del optoacoplador PC817 (hoja técnica de Sharp) al cual se incorporó una resistencia serie para limitar la corriente. El circuito de entrada se completa con un capacitor para permitir el pasaje de las altas frecuencias generadas por el inversor y con una resistencia en paralelo con los diodos para endurecer la sensibilidad evitando falsos disparos por las capacidades entre conductores de la instalación eléctrica donde se lo emplee.

La etapa de salida inicia en el fototransistor del opto-acoplador, el cual se polariza en colector mediante una resistencia a $5 \mathrm{~V}$. Como el LED del opto-acoplador es alimentado con una tensión pulsante la salida del transistor también lo será, para integrarla se utilizó un capacitor electrolítico. La respuesta de transferencia del opto-acoplador tiene una parte lineal y dado que pretendemos que la señal de nuestro detector de carga sea todo o nada se debe incorporar al circuito un discriminador que introduzca cierta histéresis para evitar falsos disparos. Esto último se logró mediante un amplificador operacional LM358 realimentado positivamente (Millman y Halkias, 1976).

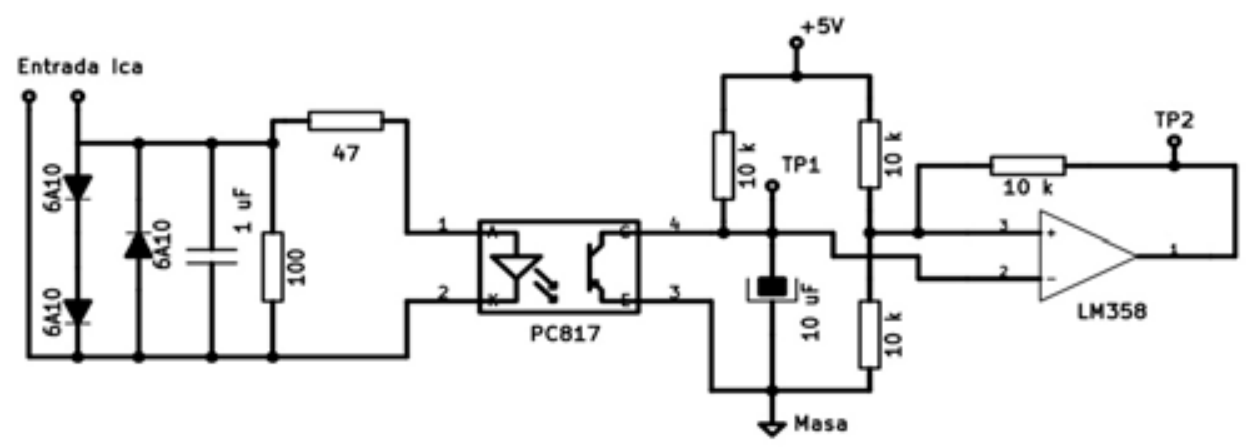

Figura 3, Circuito esquemático del detector de carga.

\section{Resultados y conclusión:}

Si bien en el presente trabajo se detalla la circuitería, el inversor a sido probado a diferentes potencias con cargas resistivas y estos datos fueron publicado en (Toranzos 2013), de la misma manera el sensor de deteccíon de carga se ensayó y publico en (Marder 2015). En esta publicación se muestra el circuito completo el cual funcionó correctamente como se planteó desde un principio lográndose un ahorro en el autoconsumo por marcha en vacío del 90\% y siendo la sensibilidad para detectar la carga de $5 \mathrm{~W}$ aproximadamente. Respecto a las prestaciones en general con cargas entre 50 y $200 \mathrm{~W}$ el inversor presentó un rendimiento superior al $80 \%$. Por lo expuesto, su uso es viable en instalaciones fotovoltaicas de baja potencia aisladas de la red eléctrica, con un desempeño similar a los productor comerciales ofrecidos por empresas internacionales pero de posible manufactura local y costo moderado. 


\section{BIBLIOGRAFIA:}

(Hoja técnica de Motorola) MOTOROLA. (1996) Switchmode Pulse Width Modulation Control Circuit TL494 Datasheet. Motorola Inc. pp. 1-12.

TEXAS INSTRUMENTS. (1999) Power Supply Control Products. Texas Instruments Inc. USA. pp. 281-289.

IEEE 602-1996 Electric Systems in Health Care Facilities. (1996) Institute of Electrical and Electronics Engineers Inc. pp. 189193.

RAMSHAW, R. (1997) Electrónica de Potencia. Marcombo, Barcelona, España. pp. 231246.

RCA Silicon Power Circuits Manual. (1967) Technical Series SP-50. USA. pp. 147-186.

Giovani, Z. Venini, C. Ferro, A. (2009) New Control Techniques for UPS Dynamic Efficiency Optimisation. Pag 1-5. Telecomunications Energy Conference, INTELEC .

Hhkachi, K. Sakane, M. Matsui, T. (1995) "Cost-Effective Practical Developments of High-Performance and Multi- Functional UPS with New System Configurations and Their Specific Control Implementations", 26th Annual IEEE Power Electronics Specialists Conference. Vol 1. Pag 480-405.

Luque A. y Hegedus S. (2011). Handbook of photovoltaic science and engineering, pp. 46-47, Wiley \& Sons Ltd.

Marder F., Toranzos V., Aquino C. y Lombardero O. (2008). Sensores lineales de tensión y corriente. Ing. Eléctrica 223, 10-13.

Millman J. y Halkias C. (1976), Integrated
Electronics: Analog and Digital Circuits and Systems. pp. 583-584 McGraw-Hill.

(hoja técnica de Diodes Inc.) . http://www.diodes.com/datasheets/ds28009.pdf

(hoja técnica de Sharp). http://www.rcscomponents.kiev.ua/datasheets/pc817s.pdf

Pompili F. (2002). Simple Circuit Measures V-I Phase Displacement. http://electronicdesign.com/test-amp-measurement/simplecircuit-measures- $v$-i-phase-displacement

(link de internet kicad). http://www. kicad-pcb.org/display/KICAD/ $\mathrm{KiCad}+\mathrm{EDA}+$ Software+Suite

(link de internet Qucs). http://qucs.sourceforge.net/

(hoja técnica inversor Magnum Energy). http://africanenergy.com/new/wp-content/ uploads/2012/08/magnummmedatasheet. pdf

(hoja de datos LM555) http://www.ti.com/lit/ ds/symlink/ne555.pdf

(hoja de datos IRFZ44) http://skory.z-net.hu/ alkatresz/irfz44.pdf

Toranzos, V. J., Aquino, C. D. J., Cáceres, M., Marder, F., \& Lombardero, O. G. (2013), Diseño de un convertidor DC/AC para equipos portátiles, Ingeniería Eléctrica 275, 34-39.

V. Marder, C. Cadena, V.Toranzos (2015), Sensor de corriente de estado sólido para detección de carga en inversores de baja potencia, aceptado para publicación congreso ASADES 2015. 